\title{
Competencia digital y alfabetización digital de los adultos (profesorado y familias)
}

Recibido: 24 de mayo de 2018 / Revisado: 5 de junio de 2018

Aceptado: 13 de junio de 2018 / Publicado: 12 de julio de 2018

DOI: $10.24310 / \mathrm{IJNE} 1.1 .2018 .4950$

\section{RESUMEN}

Los menores tienen un contacto cada vez más temprano y estrecho con las TIC, aunque con frecuencia carecen de las destrezas necesarias para usarlas de modo seguro y eficiente. En términos de alfabetización digital de los niños, los adultos juegan un papel muy importante en tres aspectos principales: como formadores o responsables directos de su alfabetización digital; como acompañantes o referentes a los que los niños pueden pedir ayuda; y como ejemplo de modelos de comportamiento. Para ejercer estos roles adecuadamente los adultos precisan de un nivel de competencia digital que debería adquirirse a través de una formación específica. Los objetivos de este estudio son: (1) identificar el nivel de alfabetización digital en una muestra de adultos (profesorado y fa-

\section{ABSTRACT}

Children have an increasingly early and close contact with ICT, although they often lack the skills necessary to use them safely and efficiently. In terms of children's digital literacy, adults play a very important role in three main aspects: as trainers or directly responsible for their digital literacy; as companions or referents to which children can ask for help; and as an example of behavior models. To exercise these roles adequately adults need a level of digital competence that should be acquired through specific training. The objectives of this study are: (1) to identify the level of digital literacy in a sample of adults (teachers and families), (2) to indicate what kind of digital competences teachers should develop, (3) indicate what type of digital competences should develop families. 
milias), (2) indicar qué tipo de competencias digitales debería desarrollar el profesorado, (3) indicar qué tipo de competencias digitales deberían desarrollar las familias. La metodología empleada se corresponde con la de un estudio de caso, para el que se emplea un cuestionario de diseño propio con 25 preguntas de respuesta dicotómica, sobre aspectos conceptuales y procedimentales (utilización del medio y gestión adecuada de la información personal). Los resultados indican que profesorado y familias muestran un nivel alto y comparable, de conocimiento de conceptos relacionados con la identidad digital, y de destrezas procedimentales básicas en el uso de redes sociales. Sin embargo, muestran un menor dominio de destrezas prácticas para la gestión de la reputación y la comunicación on line. Las conclusiones del estudio señalan la importancia de alfabetizar digitalmente a los adultos para hacerles competentes en todas las áreas identificadas en el marco común de la competencia digital docente.

PALABRAS CLAVE: Competencia digital, alfabetización digital, profesorado, familias.
The methodology used corresponds to that of a case study, for which a self-designed questionnaire is used with 25 dichotomous response questions, on conceptual and procedural aspects (use of the medium and adequate management of personal information). The results indicate that teachers and families show a high and comparable level of knowledge of concepts related to digital identity, and of basic procedural skills in the use of social networks. However, they show a lesser mastery of practical skills for reputation management and online communication. The conclusions of the study point out the importance of digitally literate adults to make them competent in all the areas identified in the common framework of digital teaching competence.

KEYWORDS: digital skills, digital literacy, teachers, families.

\section{Introducción}

En España, los menores otorgan especial importancia a las TIC. Las valoran de forma positiva y consideran "que Internet es un espacio para gestar y mejorar amistades, y que además, el teléfono móvil ofrece a los menores, libertad, intimidad, ausencia de control, espontaneidad en las expresiones, flexibilidad en los planes de actuación y encuentro con otros, etc." (Ortega-Ruiz, 2012, p.47). A ello hay que sumarle el hecho de que: "El acceso a las TIC se está produciendo a edades cada vez más tempranas. El 30\% de los niños/as españoles de 10 años de edad tiene un teléfono móvil" (Protégeles, 2014,p.3). Efectivamente, según indican datos del Instituto Nacional de Estadística (INE, 2015) y del Instituto Nacional de Tecnologías de la Comunicación (ITC, 2014), el uso de las TIC por parte de los menores (población infantil de 10 a 15 años) es elevado y aumenta cada año. De la consulta de los datos que ofrece el INE, la evolución de los resultados según la edad sugiere que el uso de Internet es una práctica mayoritaria 
antes incluso de los 10 años. La Asociación para la Investigación de Medios de Comunicación (AIMC) ofrece datos sobre el uso de internet entre los menores. Se observa que entre los niños y niñas de 4 a 13 años ha habido un incremento exponencial de la penetración de Internet: de un 57.4\% en 2012 (AIMC, 2012) a un 62.4\% en 2017 (AIMC, 2017).

Datos de diferentes estudios (Echeburúa y Requesens, 2012; Inteco, 2009 y 2011; Livingstone, Haddon, Görzig y Ólfsson, 2010) indican que los usuarios adquieren gran destreza en el uso de la tecnología, del medio, pero no tanta en el uso seguro de la misma. Niños y niñas menores de edad se exponen a diario a través de la red: gestionan su identidad digital personal, construyen su visibilidad, configuran su reputación, y definen su privacidad. Estar en el ciberespacio significa tener una representación de uno mismo, una identidad digital que se va construyendo a partir de la propia actividad en Internet y de la actividad de los demás (Gionés-Valls y Serrat-Brustenga, 2010). Estas informaciones, mal gestionadas, suponen el desarrollo de conductas de riesgo por parte de los sujetos. Son conductas que pueden implicar o derivar en situaciones problemáticas de distintas naturaleza y alcance como las relacionadas con el sexting, el grooming, o el ciberacoso entre otras. Como señalan Ortega-Ruiz, Del Rey y Sánchez (2012) los riesgos a los que se enfrentan los niños y adolescentes en Internet vienen a veces provocados por el uso inadecuado o excesivo de las nuevas tecnologías. Otras muchas veces por la poca educación que han recibido en el uso responsable de las mismas, y muchas otras por la poca supervisión que los adultos (la familia) ejercen con los hijos. En este sentido Castro (2013) afirma que los niños y adolescentes que navegan en Internet en casa sin un adecuado control parental, están totalmente expuestos a todo tipo de riesgos. El autor indica que es necesario que tengan unos límites, y que sean educados en un uso responsable de las TIC.

Es, por tanto, necesario, asegurar la alfabetización digital de niños y niñas, entendida esta como preparación para desenvolverse en una cultura digital, o desarrollo de la competencia digital. Para la infancia y la juventud las TIC no sólo son objetos normales de su paisaje vital y de su experiencia cotidiana, son también una seña de identidad generacional, que los distingue del mundo de los adultos (Area, 2014, p.8). Se convierten en consumidores de información masiva e indiscriminada de información. También en creadores y diseminadores de nueva y libre información. En todo ello debe mediar una adecuado proceso de alfabetización digital que permita aprender a manejar los aparatos y su software, pero que permita también desarrollar competencias o habilidades cognitivas relacionadas con la obtención, comprensión y elaboración de la información (Area y Guarro, 2012). Según indica el autor, la alfabetización digital centra su atención en la adquisición y dominio de destrezas centradas en el uso de la información y la comunicación, y no tanto en las habilidades de utilización de la tecnología. Queda claro que, como señalan López-Sánchez, y García del Castillo (2016) los niños y jóvenes son vulnerables ante las TIC, y que se hace necesaria para ellos la supervisión de los adultos de referencia, para que actúen como factores de protección, e intervengan como educadores y formadores de su aprendizaje. 
La familia es clave en el necesario empoderamiento de los más jóvenes en el uso de las TIC tal y como indican Torrecillas-Lacave, Vázquez-Barrio y Monteagudo-Barandalla (2017). Pero son diversos los autores que señalan que las familias desean recibir formación que les permita acceder a una adecuada alfabetización digital (Aguilar-Ramos y Urbano, 2014). Porque como indica la realidad que señalan Plaza y Caro (2016), muchos adultos (padres y profesores) no son capaces de orientar a los niños y jóvenes en los códigos y el uso responsable de las TIC. Una de las razones es que, como describen GarridoLora, Busquet-Durán y Munté-Ramos (2016), se ha producido una inversión del proceso educativo. Esta inversión consiste en que si en la primera mitad del siglo XX eran lo adultos quienes transmitían los conocimientos y el mapa de experiencia necesarios, ahora son los jóvenes quienes poseen un mejor conocimiento de los nuevos códigos (Plaza y Caro, 2018). Se habla así como lo hacen Garrido-Lora et al. (2016) de la existencia de una "brecha digital generacional", que se pone de manifiesto por "la existencia de evidentes diferencias entre generaciones tanto en el conocimiento como en el uso de las TIC y las redes sociales" (p.52). Los adultos (tanto la familia como la escuela) deben adquirir las competencias de la nueva alfabetización que les permitan superar la brecha digital e inter-generacional (Aguilar-Ramos y Urbano, 2014).En este estudio, para definir el concepto de alfabetización digital que se emplea, se toma como referencia dos definiciones: (1) la de Area y Pessoa (2012) en su Modelo de Alfabetización Integrado (Area y Pessoa, 2012; Area y Guarro, 2012; Area, 2014; Area et al., 2015); (2) la de Gionés-Valls y Serrat-Brustenga (2010) sobre cómo configurar la identidad personal en el mundo digital (Fernández-Montalvo, Peñalva-Vélez e Irazabal, 2015; Fernández-Montalvo, Peñalva-Vélez, Irazabal y López-Goñi, 2017).

En primer lugar, a través del modelo de alfabetización de Area y Pessoa (2012) se indica que las dimensiones de la Web 2.0 en la que los sujetos deben alfabetizarse para poder hacer un uso seguro de Internet son seis. Estar alfabetizado va a implicar por lo tanto, saber usar la web 2.0 como: (1) una biblioteca universal, (2) un mercado de servicios, (3) un puzzle de microcontenidos entrelazados, (4) un espacio público de comunicación en redes sociales, (5) un espacio de expresión multimedia y audiovisual, (6) un territorio de experiencias virtuales interactivas. En segundo lugar, en base a lo desarrollado por Gionés-Valls y Serrat-Brustenga (2010) se aborda la alfabetización digital como el proceso de enseñar a cómo usar de manera segura Internet, a través de una adecuada configuración de la identidad personal en el mundo digital. Entendiendo la identidad digital personal como la habilidad para gestionar con éxito la propia visibilidad, reputación y privacidad en la red. Ambas definiciones sobre qué es la alfabetización digital inciden en un aspecto básico de las TIC, el relativo a su uso como herramientas de comunicación y gestión de la información (Blasco y Durban, 2012; Gutiérrez, 2010). Ambas se refieren al uso eficaz de Internet no tanto para saber desenvolverse a nivel instrumental en el medio, como por saber gestionar de un modo seguro todos los datos que se transmiten y reciben a través de él (De Pablos, 2010). 
En este contexto, como ya se ha señalado, el papel de los adultos resulta determinante a la hora de promover actitudes y conocimientos en los menores, para que hagan un uso seguro de Internet, y para que adquieran destrezas en el acceso a la información y uso adecuado de la misma (Fernández-Montalvo et al., 2017; Peñalva-Vélez, Leiva e Irazabal, 2017). Resulta fundamental "que todo el mundo (padres, tutores, profesores, instituciones y gobiernos) trabajen conjuntamente para crear entornos seguros y accesibles para los niños y los jóvenes, estén donde estén: en casa, en la escuela o en instalaciones públicas" (Internet Society, 2012, p.2). En términos de alfabetización digital de los niños, los adultos juegan un papel muy importante en tres aspectos principales: (1) como formadores, como responsables directos de su alfabetización digital; (2) como acompañamiento, como referentes a los que los niños pueden pedir ayuda; y (3) como ejemplo de modelos de comportamiento (Peñalva et al., 2017). La escuela se enfrenta al reto de contribuir a promover la necesaria alfabetización mediática y digital (Aguilar-Ramos y Urbano, 2014) y al de disminuir la distancia digital entre adultos y jóvenes (GarridoLora et al., 2016). La familia comparte con el profesorado el reto de disminución de esta brecha digital (Garrido-Lora et al., 2016), y se enfrenta a otros como los de asumir su papel como empoderadora de los niños y niñas en el uso seguro y crítico de Internet (Plaza y Caro, 2018; Sánchez-Valle, De FrutosTorres y Vázquez-Barrio, 2017).

Resulta innegable que, para hacer frente a estos retos, los adultos deben poseer también un cierto nivel de competencia digital. Por todo esto los objetivos de este estudio son dos: (1) indagar sobre el nivel de alfabetización digital que presenta una muestra de profesorado y familias. Para ello se plantea un estudio a través del que se obtiene información sobre aspectos concretos de la alfabetización digital que muestran los sujetos de la muestra a nivel conceptual y a nivel procedimental (en el nivel instrumental y en el nivel de gestión de la identidad digital). (2) Identificar los elementos que debería incluir un plan de formación digital para maestros y familias, partiendo de los puntos fuertes y débiles reflejados por los sujetos de ambas muestras. Porque "se hace más necesario que nunca que los adultos retomen el papel de referentes en esta cuestión, ya que son muchos los menores de edad que manifiestan su necesidad de recibir formación e información" (Protégeles, 2014, p.20).

\section{Material y método}

Para la realización de este estudio se ha empleado un instrumento formado por un listado de 28 preguntas. Los tres primeros ítems son de respuesta múltiple e identifican los datos sociodemográficos de la muestra referidos al sexo, la edad y el curso en el que se imparte docencia o al que pertenecen los hijos o hijas. Los 25 ítems restantes incluyen cuestiones referidas al nivel de alfabetización digital conceptual (Sabes qué es...; 10 ítems) y al nivel de alfabetización digital procedimental (Sabes cómo se hace...; 15 ítems). A su vez, en en este nivel se distingue entre aquellas conductas que implican saber 
cómo desenvolverse a nivel instrumental en el medio ( 8 items) y entre aquellas conductas que implican saber cómo gestionar de un modo seguro los datos que se transmiten y reciben ( 7 items). Los ítems son de respuesta dicotómica (si/no).

La muestra de este estudio está formada por 30 profesores y profesoras de los cursos $3^{\circ}$ a $6^{\circ}$ de Educación Primaria (un 90\% mujeres y un 10\% hombres). La muestra de familias está formada por un total de 42 padres y madres (un 78,6\% mujeres y un 21,4\% hombres) con hijos e hijas escolarizados en los cursos $3^{\circ}$ a $6^{\circ}$ de Educación Primaria. La muestra forma parte de un estudio más amplio sobre los niveles de alfabetización digital de una muestra de niños y niñas de $3^{\circ}$ a $6^{\circ}$ de Educación Primaria. En dicho estudio se pretende definir los patrones de uso de Internet y del teléfono móvil que tienen los niños y niñas, analizando de forma paralela la alfabetización digital que muestran los adultos responsables de su seguridad en el uso que hacen de Internet (Protégeles, 2014; Fernández-Montalvo et al., 2015; Fernández-Montalvo et al., 2017).

\section{Resultados}

El análisis de los datos referidos a las tres categorías de alfabetización manifestada por la muestra indica que tanto maestros como familias muestran un nivel alto de alfabetización digital conceptual. Es decir, en esta primera categoría la mayoría de los sujetos de la muestra conocen los conceptos presentados, relacionados con la configuración de la identidad digital (Gráfico 1). En ambas muestras (profesorado y familias) se observa que los sujetos manifiestan conocer aquellos conceptos que se manejan de forma más habitual (bullying, ciberbullying, datos personales, o privacidad en Internet), y que tienen que ver con las conductas de riesgo que más comúnmente se ponen en marcha en la Red. Sin embargo conceptos como los de identidad digital, identidad analógica, reputación o visibilidad en internet, son menos conocidos por los sujetos., pese a ser los conceptos que favorecen una gestión segura de la información y las conductas propias y ajenas en la Red. El nivel de alfabetización es muy homogéneo entre la muestra de profesores y familias.

Cuando se aborda la segunda categoría de análisis relativa al nivel de alfabetización procedimental (en el nivel instrumental), profesorado y familias muestran un buen nivel en lo referido a aquellas conductas que implican saber cómo desenvolverse en el medio. No hay grandes diferencias entre muestras, y únicamente destaca el hecho que ninguno de los dos segmentos conozca cómo eliminar datos colgados en la Red (53-60\%) (Gráfico 2).

Respecto a la tercera y última categoría, relativa también al nivel de alfabetización procedimental pero referido a la gestión de la identidad, se observa que el nivel de alfabetización de los adultos baja (Gráfico 3). 
¿Sabes qué es...?
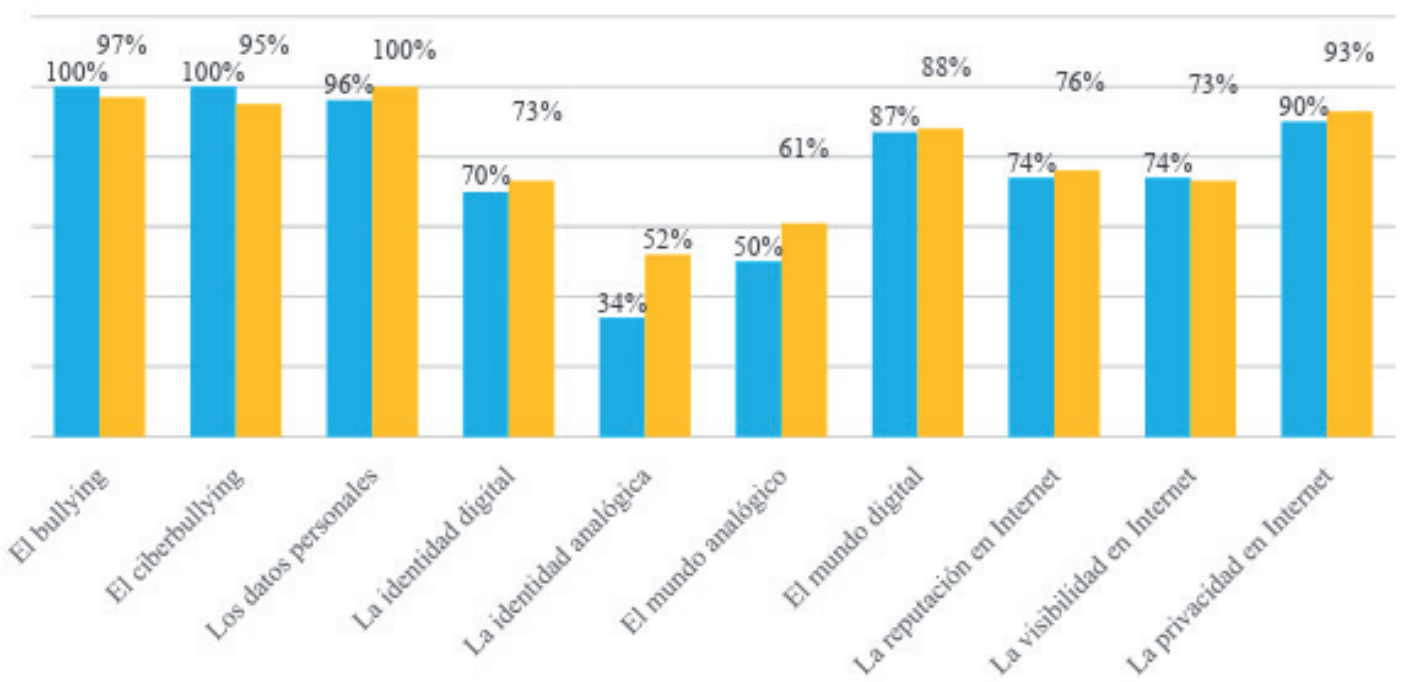

Profesorado Familias

Gráfico 1. Nivel de alfabetización digital conceptual de familias y profesorado

¿Sabes cómo...?

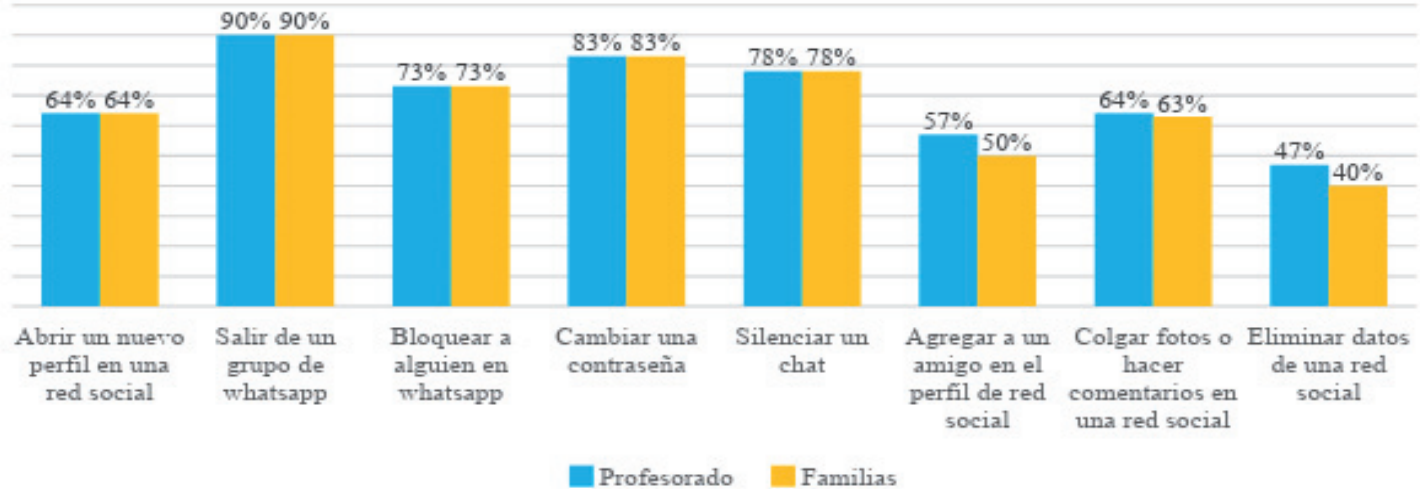

Gráfico 2. Alfabetización digital procedimental (nivel instrumental).

Cuando se trata de poner en marcha aquellas acciones que ayudan a gestionar de un modo seguro la identidad en la Red se observan diferencias poco significativas entre las muestras. Con todo, parece ser la muestra de profesorado la que se percibe más capaz de poner en marcha aquellas conductas que implican saber cómo gestionar de un modo seguro los datos que se transmiten y reciben. En este caso, las familias muestran un nivel de alfabetización inferior al del profesorado. Destaca el hecho de que 
la configuración de la reputación en Internet sea algo que la gran mayoría de la muestra de familias (95\%) desconoce, al igual que un importante porcentaje de la muestra de profesorado (67\%).

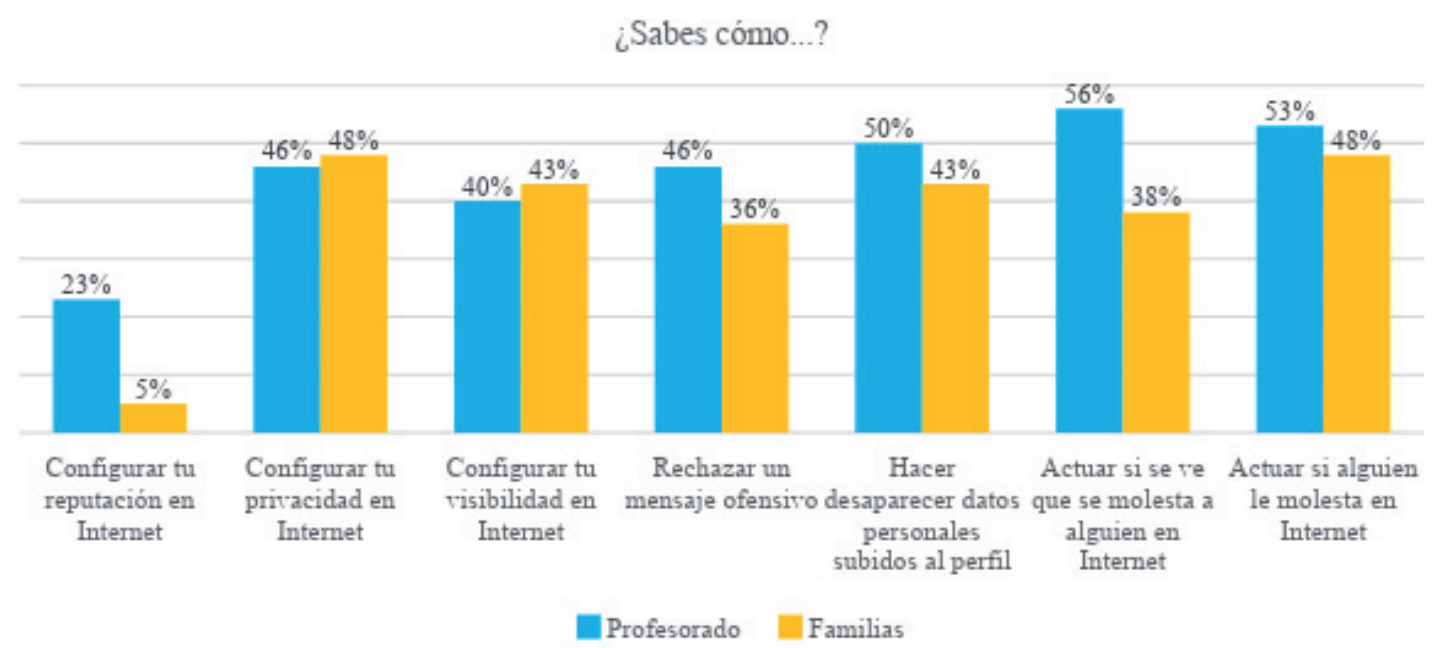

Gráfico 3. Alfabetización digital procedimental (gestión de la identidad).

\section{Discusión y conclusiones}

A la vista de los datos obtenidos se puede observar que los adultos de la muestra presentan un alto grado de alfabetización digital a nivel conceptual. Conocen algunos de los principales conceptos relacionados con las dimensiones de la web 2.0 identificadas por Area y Pessoa (2012). Conocen en mayor o menor medida los conceptos vinculados al uso seguro de Internet y a la configuración de la Identidad digital. Se constata que por el contrario, en nivel de alfabetización digital de los adultos desciende cuando se trata del ámbito procedimental. A la hora de poner en marcha aquellas conductas y acciones que permiten configurar de manera activa el uso seguro de Internet, los adultos se muestran competentes para saber desenvolverse a nivel instrumental en el medio, pero no muy competentes para saber gestionar de un modo seguro los datos que transmiten y reciben.

Dado que tanto las familias como el profesorado son los adultos de referencia para los niños y jóvenes a la hora de empoderarse digitalmente, de alfabetizarse digitalmente, no se debe perder de vista su formación en este sentido. La familia desde el ámbito privado, en los contextos principalmente de ocio y de expresión lúdica. El profesorado desde el ámbito más académico, en los contextos de aprendizaje formal y de incorporación de las TIC a la escuela. Todos ellos desempeñan roles fundamentales en el proceso de socialización de los niños y jóvenes a través de las TIC, y en el proceso de aprendizaje del 
medio que deben desarrollar: alfabetización mediática y digital. Que los adultos estén o no formados tiene un efecto práctico directo (e indeleble) sobre la identidad que niños y jóvenes construyen en el medio digital. Resulta imperativo, según los datos presentados, que se formen en ámbitos específicos como:

- la gestión adecuada de la identidad digital: y en consecuencia, de la privacidad (con la protección activa de los datos personales propios y ajenos), de la visibilidad (con la generación de actividades positivas en la red) y de la reputación (con la construcción de una huella digital adecuada) (Gionés-Valls y Serrat-Brustenga, 2010).

- la puesta en marcha de relaciones interpersonales positivas en los contextos virtuales (OrtegaRuiz, Casas y Del Rey, 2014): a través de pautas o "normas de etiqueta en la Red" como las de la netiqueta joven para redes sociales (Flores, 2010; Castillejos, Torres y Lagunes, 2016).

- el conocimiento de la web 2.0 y sus dimensiones: biblioteca, mercado de servicios, puzzle de contenidos, espacio público de comunicación, escenario de expresión multimedia, entorno de experiencias virtuales (Area y Pessoa, 2012).

- el conocimiento de las herramientas del web 2.0 y su aplicación a la web 3.0 o web semántica (Moreno, 2015; Pérez-Tornero y Tejedor, 2016).

La formación o alfabetización digital de profesorado y familias forma parte de los objetivos de la educación para la década 2010-2020 propuestos desde el Ministerio de Educación según indican Aguilar-Ramos y Urbano (2014). Como señalan distintos autores, las políticas educativas han realizado evidentes esfuerzos por introducir las TIC en los centros escolares (Aguilar y Leiva, 2012; Linde y Ramos, 2014; Area, Sanabria y Vega, 2015). La competencia digital, según la Ley Orgánica 8/2013 de Mejora de la Calidad Educativa, implica el uso creativo, crítico y seguro de las tecnologías de la información y la comunicación para alcanzar los objetivos relacionados con el trabajo, la empleabilidad, el aprendizaje, el uso del tiempo libre, la inclusión y participación en la sociedad.

Requiere conocimientos técnicos, pero también el desarrollo de diversas destrezas relacionadas con (1) el acceso a la información, (2) el procesamiento y uso para la comunicación, (3) la creación de contenidos, (4) la seguridad y (5) la resolución de problemas, tanto en contextos formales como no formales e informales. La adquisición de esta competencia requiere además actitudes y valores que permitan al usuario adaptarse a las nuevas necesidades establecidas por las tecnologías, su apropiación y adaptación a los propios fines y la capacidad de interaccionar socialmente en torno a ellas. Por otra parte, la competencia digital implica la participación y el trabajo colaborativo, así como la motivación y la curiosidad por el aprendizaje y la mejora en el uso de las tecnologías. A los efectos de este estudio, interesa destacar las necesidades formativas de la muestra, y la necesidad de alfabetización digital de los adultos constata- 
da por los diferentes autores. Todo ello para proponer que dichas necesidades se planteen en el marco de las cinco áreas que hace define el INTEF (2017), en su Marco Común de Competencia Digital Docente, concretando y desarrollando los conceptos clave identificados en el proyecto DigiComp (Ferrari, 2013).

\section{Referencias}

Aguilar, M.C. y Leiva, J.J. (2012). La participación de las familias en las escuelas TIC: análisis y reflexiones educativas. Píxel-Bit. Revista de Medios y Educación, (40), 7-19.

Aguilar-Ramos, M.C. y Urbano, A. (2014). La necesidad de alfabetización digital e inter-generacional en la familia y la escuela. Didáctica, Innovación y Multimedia (DIM), 28, 1-16.

AIMC-Asociación para la intestigación de medios de comunicación (2012). Resumen General de resultados EGM ( $1^{\text {a }}$ ola del estudio general de medios). Recuperado de https://www. aimc.es/a1mc-c0nt3nt/uploads/2012/05/Acceso Internet ninos menores 14 anos en EGM-2.pdf

AIMC-Asociación para la intestigación de medios de comunicación (2018). Resumen General de resultados EGM (2 $2^{\text {a }}$ ola del estudio general de medios) abril 2017 a marzo 2018. Recuperado de https://www.aimc.es/a1mc-c0nt3nt/uploads/2018/04/resumegm118.pdf

Área, M. (2014). Alfabetización digital y competencias profesionales para la información y la comunicación. Organización y gestión educativa: Revista del Fórum Europeo de Administradores de la Educación, 22 (1), 9-13.

Area, M. y Guarro, A. (2012). Revista Española de Documentación Científica, Monográfico, 46-74.

Área, M., Borrás, J.F. \& San Nicolás, B. (2015). Educar a la generación de los Millenials como ciudadanos cultos del ciberespacio. Apuntes para la alfabetización digital. Revista de Estudios de Juventud, 109, 13-32.

Area, M., y Pessoa, T. (2012). De lo sólido a lo líquido: las nuevas alfabetizaciones ante los cambios culturales de la Web 2.0. Comunicar, 38, 13-20.

Area, M., Sanabria, A.L. y Vega, A.M. (2015). Las políticas educativas TIC (Escuela 2.0) en las Comunidades Autónomas de España desde la visión del profesorado. Campus virtuales, 2(1), 74-88.

Blasco, A. y Durban, G. (2012). Competència informacional: del currículum a l'aula. Barcelona: Rosa Sensat. 
Castillejos, B., Torres, C. A. y Lagunes, A. (2016). La seguridad en las competencias digitales de los millennials. Apertura (Guadalajara, Jal.), 8(2), 54-69.

Castro, A. (2013). Formar para la ciberconvivencia Internet y prevención del ciberbullying. Revista Integra Educativa, 6(2), 49-70.

De Pablos, J. (2010). Universidad y sociedad del conocimiento. Las competencias informacionales y digitales. En: Competencias informacionales y digitales en educación superior [monográfico en línea]. Revista de universidad y sociedad del conocimiento (RUSC), 7(2).doi http://dx.doi.org/10.7238/rusc.v7i2.977 Disponible en http://www.temoa.info/ es/node/47969

Echeburúa, E. y Requesens, A. (2012). Adicción a las redes sociales y nuevas tecnologías en niños y adolescentes. Guía para educadores. Madrid: Pirámide.

Fernandez-Montalvo, J., Peñalva Vélez, A., \& Irazabal, I. (2015). Internet use habits and risk behaviours in preadolescence. Comunicar, 44, vol. XXII, pp. 113-120.

Fernández-Montalvo, J., Peñalva Vélez, A., Irazabal, I., \& López-Goñi, J. J. (2017). Efectividad de un programa de alfabetización digital para estudiantes de Educación Primaria. Cultura y Educación (2017), 29 (1), 1-30.

Ferrari, A. (2013). DIGCOMP: A framework for developing and understanding digital competence in Europe.

Flores, J. (2010). La netiqueta joven para redes sociales. Recuperado de http://www.netiquetate. $\underline{\mathrm{com} /}$

Garrido-Lora, M.; Busquet-Durán, J. y Munté-Ramos, R. A. (2016). De las TIC a las TRIC. Estudio sobre el uso de las TIC y la brecha digital entre adultos y adolescentes en España. Anàlisi. Quaderns de Comunicació i Cultura, 54, págs. 44-57.

Gionés-Valls, A. y Serrat-Brustenga, M. (2010). La gestión de la identidad digital: una nueva habilidad informacional y digital. BiD: textos universitaris de biblioteconomia i documentació, 24, 1-15.

Gutiérrez, A. (2010). Creación multimedia y alfabetización en la era digital. En Aparici, R.(coord.). Educomunicación: más allá del 2.0. Barcelona: Gedisa. 
INE-Instituto Nacional de Estadística (2015). Encuesta sobre equipamiento y uso de tecnologías de información y comunicación en los hogares (TIC-H). Madrid: Instituto Nacional de Estadística. Recuperado el 2 de febrero de 2016 de: http://www.ine.es/prensa/np933.pdf

Inteco (2009). Estudio sobre hábitos seguros en el uso de las TIC por niños y adolescentes y econfianza de sus padres. Recuperado el 24 de febrero de 2016 en http://www.pantallasamigas.net/actualidad-pantallasamigas/pdf/inteco-estudio-uso-seguro-tic-menores.pdf

Inteco (2011). Estudio sobre seguridad y privacidad en el uso de los servicios móviles españoles. Recuperado de http://www.pantallasamigas.net/pdf/estudio sobre seguridad y privacidad en el uso de los servicios moviles por los menores espanoles.pdf

INTEF, 2017. Marco Común de Competencia Digital Docente. Disponible en 07/04/2018 en http:// educalab.es/documents/10180/12809/MarcoComunCompeDigiDoceV2.pdf

ITC-Instituto Nacional de Tecnologías de la Comunicación (2014). Estudio sobre la ciberseguridad y confianza en los hogares españoles.

Linde, T. y Ramos, M. C. (2014). Formación inicial innovadora del profesorado hacia el desarrollo de competencias y alfabetización digital. Congreso Internacional EDUTEC.

Livingstone, S., Haddon, L., Görzig, A., Ólfsson, K. (2010). Risks and safety on the Internet: The perspective of European children. Initial Findings. LSE, London: EU Kids On-line.

López-Sánchez, C. y García del Castillo, J. A. (enero-junio, 2017). La familia como mediadora ante la brecha digital: repercusión en la autoridad.Revista Colombiana de Ciencias Sociales, 8(1), pp. 108-124. DOI: http://dx.doi.org/10.21501/22161201.1928

Moreno, N.M. (2015). Manual docente sobre recursos didácticos multimedia. Escenarios formativos virtuales. Madrid: Editorial Académica Española.

Ortega-Ruiz, R. (Dir.) (2012). Nuevas dimensiones de la convivencia escolar y juvenil. Ciberconducta y relaciones en la Red: Ciberconvivencia. Madrid:MEC. Recuperado de https:// www.uco.es/laecovi/img/recursos/p5xqp1s849A8yPq.pdf

Ortega-Ruiz, R., Casas, J.A. \& Del Rey, R. (2014). Hacia el constructode ciberconvivencia. Infancia y Aprendizaje, 37 (3), 602-628. 
Ortega-Ruiz, R., Del Rey, R. y Sánchez, V. (2012). Nuevas dimensiones de la convivencia escolar y juvenil. Ciberconducta y relaciones en la Red: Ciberconvivencia. Madrid: Observatorio Estatal de la Convivencia Escolar.

Peñalva-Vélez, A., Leiva, J.J. e Irazabal, I. (2017). The role of adults in children digital literacy. Procedia-Social and Behavioral Sciences, 237, 887-892.

Pérez-Tornero, J. M. y Tejedor, S. (Eds.). (2016). Ideas para aprender a aprender: manual de innovación educativa y tecnología. Barcelona, ESPAÑA: Editorial UOC.

Plaza, J. y Caro, C. (2016). La implicación de la familia en la formación ético-cívica de los jóvenes a través de las TIC. Aloma, 34 (2), 97-106.

Plaza, J. y Caro, C. (2018). La familia, agente clave de empoderamiento juvenil en la sociedad TIC. Cultura y Educación, 30 (2), 338-367.

Protégeles (2014). Menores de edad y conectividad móvil en España: tablets y smartphones. Recuperado de https://kidsandteensonline.files.wordpress.com/2014/07/estudio movil smartphones tablets protegeles.pdf.

Sánchez-Valle, M., De Frutos-Torres, B. y Vázquez-Barrio, T. (2017). La influencia de los padres en la adquisición de habilidades críticas en Internet. Comunicar, 25(53), 103-111.

Torrecillas-Lacave, T., Vázquez-Barrio, T., \& Monteagudo-Barandalla, L. (2017). Percepción de los padres sobre el empoderamiento digital de las familias en hogares hiperconectados. El profesional de la información (EPI), 26(1), 97-104. 